\title{
The quantum mechanical geometric phase of a particle in a resonant vibrating cavity
}

\author{
K. W. Yuen, H. T. Fung, K. M. Cheng, M. -C. Chu, and K. Colanero \\ Department of Physics, The Chinese University of Hong Kong, Shatin, N.T., Hong Kong.
}

\begin{abstract}
We study the general-setting quantum geometric phase acquired by a particle in a vibrating cavity. Solving the two-level theory with the rotating-wave approximation and the $\mathrm{SU}(2)$ method, we obtain analytic formulae that give excellent descriptions of the geometric phase, energy, and wavefunction of the resonating system. In particular, we observe a sudden $\pi$-jump in the geometric phase when the system is in resonance. We found similar behaviors in the geometric phase of a spin- $1 / 2$ particle in a rotating magnetic field, for which we developed a geometrical model to help visualize its evolution.
\end{abstract}

Typeset using REVTEX 


\section{INTRODUCTION}

The dynamics of confined cavity fields interacting with the cavity wall is of great interest for the understanding of a variety of problems such as hadron bag models [1], sonoluminescence [2], cavity QED [3] and black hole radiations [4]. Most previous studies on dynamical cavity concentrated on scalar or photon fields [5], which despite the apparent simplicity, exhibit rich and complex dynamics. In contrast, the system of a Schrödinger particle in an oscillating spherical cavity has not gained as much attention. In this article, we study the generalized quantum geometric phase of a particle in resonance with the vibrating cavity. We will show that the geometric phase acquires sudden $\pi$-jumps when the particle makes transitions.

The geometric phase of a quantum system has drawn much attention since it was first proposed by Berry in 1984 [6,7]. It reveals the geometric structure of the Hilbert space of the system $[7,8]$, and its existence in physical systems has been verified in many experiments [9], including electron diffraction from a screw dislocation [10] and neutron interferometry [11]. The phase effects in molecular physics can lead to energy splittings and shift quantum numbers. The geometric phase has also been shown to be intimately connected to the physics of fractional statistics, the quantized Hall effect, and anomalies in gauge theory [7]. As far as we know, our study represents the first calculation of the geometric phase of a resonating system, which evolves non-adiabatically and non-cyclically.

\section{FORMALISM}

We consider an infinite cylindrical or spherical potential well with oscillating boundary $[12]:$

$$
V(r)=\left\{\begin{array}{cl}
0, & \text { if } r<R(t) \\
\infty, & \text { if } r \geq R(t)
\end{array}\right.
$$

where $R(t)=R_{0} / \alpha(t)$, with $\alpha(t) \equiv[1+\epsilon \sin (\omega t)]^{-1}$. The coordinates can be transformed to a fixed domain via $\vec{y} \equiv \alpha(t) \vec{r}$, and, to preserve unitarity, the wavefunction is renormalized through $\phi(\vec{y}, t) \equiv \alpha^{-\xi} \psi(\vec{r}, t)$, where $\xi=1(3 / 2)$, for a cylindrical (spherical) well.

Since the full Hamiltonian $H(t)$ commutes with $L^{2}$ and $\vec{L}$, the wavefunction can be factorized: $\phi(\vec{y}, t)=Y(y, t) \Theta$, where $\Theta$ depends only on the angular variables. Inside the cavity, the radial wavefunction satisfies

$$
\frac{\partial Y}{\partial t}=\left[\frac{i \hbar \alpha^{2}}{2 \mu}\left(\frac{\partial^{2}}{\partial y^{2}}+\frac{n_{d}}{y} \frac{\partial}{\partial y}-\frac{m_{d}}{y^{2}}\right)+\frac{\dot{R}}{R}\left(y \frac{\partial}{\partial y}+\xi\right)\right] Y,
$$

where $\mu$ is the particle mass, and $n_{d}=1,2$ and $m_{d}=m^{2}, l(l+1)$ for cylindrical and spherical wells respectively. In this paper, we only consider the $m_{d}=0$ sector.

The wavefunction described by Eq. 2 evolves in time and acquires a time-dependent phase, which in general consists of a dynamical phase and a geometric one [6]. When the dynamics is adiabatic and/or the evolution is cyclic, the geometric phase, or Berry's phase, has been studied for many systems. Since we are interested in the geometric phase of a nonadiabatic, non-cyclic system, we have to resort to a generalized method. Following Ref. [13], 
we first remove the dynamical phase from the wavefunction of the system: $|\phi(t)\rangle \rightarrow|\tilde{\phi}(t)\rangle \equiv$ $e^{-i \theta(t)}|\phi(t)\rangle$, the dynamical phase is

$$
\theta(t) \equiv-\frac{1}{\hbar} \int_{0}^{t} E\left(t^{\prime}\right) d t^{\prime}
$$

where $E\left(t^{\prime}\right)=\operatorname{Re}\left\langle\phi\left(t^{\prime}\right)\left|H\left(t^{\prime}\right)\right| \phi\left(t^{\prime}\right)\right\rangle$.

The general setting geometric phase, or the Pancharatnam phase $\beta$ is defined as the relative phase between the state at time $t_{1}$ and that at time $t$, both with the dynamical phase removed. It can be obtained from the inner product of these two states

$$
\beta\left(t_{1}, t\right) \equiv-i \log \left[\frac{\left\langle\tilde{\phi}\left(t_{1}\right) \mid \tilde{\phi}(t)\right\rangle}{\left|\left\langle\tilde{\phi}\left(t_{1}\right) \mid \tilde{\phi}(t)\right\rangle\right|}\right] .
$$

Our main goal in this paper is to study this geometric phase for a simple but nontrivial dynamical system.

\section{RESULTS}

We have solved Eq. 2 numerically and checked that the solution is stable and converges very well. In Fig. 1, we show the maximum energy of the particle as a function of the

driving frequency $\omega$, both having been scaled to be dimensionless: $\tilde{E}_{\max } \equiv \mu R_{0}^{2} E_{\max } / \hbar^{2}$, and $\tilde{\omega} \equiv \mu R_{0}^{2} \omega / \hbar$. The particle is initially in the ground state, and it is in resonance at specific values of $\tilde{\omega}$. In each period of cavity vibration $\tau \equiv 2 \pi / \tilde{\omega}$, the geometric phase acquires some changes, $\beta_{1}\left(t_{1}\right) \equiv \beta\left(t_{1}, t_{1}+\tau\right)$, and it exhibits oscillations at these resonances. We show three examples in Fig. 2 for $\tilde{\omega}=66.632$, 17.278 and 22.21227, where the geometric phase acquires periodic changes of $2 \pi, 4 \pi, 6 \pi$ respectively. All the resonances shown in Fig. 1 are associated with oscillations of $\beta_{1}$ with amplitudes of $2 N \pi, N=1,2,3$.

To understand the resonances and the associated geometric phases, we limit ourselves to the parameter regime where the Hamiltonian can be truncated to a two-level system. Specifically, if $\epsilon<<1$, and the initial state is the $k^{\text {th }}$ unperturbed eigenstate $\left|\phi_{k}(y)\right\rangle$, with eigenenergy $E_{k}$, then when the driving frequency corresponds to the energy difference between the initial state and another unperturbed eigenstate $\left|\phi_{n}(y)\right\rangle$, with eigenenergy $E_{n}$, i.e., $\omega=\omega_{n k} \equiv\left(E_{n}-E_{k}\right) / \hbar$, the particle is expected to behave as in a two-level system. Then the problem simplifies considerably, and we have obtained its solution with two analytic approaches: the SU(2) method and the Rotating-wave Approximation (RWA).

Following Cheng et al. [14] we first expand the time-dependent Hamiltonian $H(t)$ of a two-level system in the identity operator $I$, the raising and lowering operators $\sigma_{ \pm}$, and Pauli spin matrix $\sigma_{3}$

$$
H(t)=i\left[f_{0}(t) I+f_{1}(t) \sigma_{+}+f_{2}(t) \sigma_{3}+f_{3}(t) \sigma_{-}\right]
$$

where $f_{j}, j=0,1,2,3$, are in general complex functions of time. The evolution operator can then be written as

$$
U(t, 0)=b(t) e^{\int_{0}^{t} f_{0}\left(t^{\prime}\right) d t^{\prime}}\left(\begin{array}{cc}
b^{-2}(t)+g_{1}(t) g_{3}(t) & g_{1}(t) \\
g_{3}(t) & 1
\end{array}\right),
$$


where $b(t) \equiv \exp \left(-g_{2}(t)\right)$, and $g_{i}$ satisfies

$$
\begin{aligned}
& \dot{g_{1}}=f_{1}+2 f_{2} g_{1}-f_{3} g_{1}^{2}, \\
& \dot{g}_{2}=f_{2}-f_{3} g_{1}, \\
& \dot{g_{3}}=f_{3} b^{-2},
\end{aligned}
$$

with the initial conditions $g_{j}(0)=0$ for $j=1,2$, or 3 .

Suppose that the two levels in which the system oscillates are $\left|\phi_{k}(y)\right\rangle$ and $\left|\phi_{n}(y)\right\rangle$, so that the wavefunction is $|\phi(y, t)\rangle=a_{k}(t)\left|\phi_{k}(y)\right\rangle+a_{n}(t)\left|\phi_{n}(y)\right\rangle$. Furthermore, if the initial conditions are $a_{k}(0)=1$ and $a_{n}(0)=0$, then

$$
\left(\begin{array}{c}
a_{k}(t) \\
a_{n}(t)
\end{array}\right)=b(t) e^{\int_{0}^{t} f_{0}\left(t^{\prime}\right) d t^{\prime}}\left(\begin{array}{c}
b^{-2}+g_{1} g_{3} \\
g_{3}
\end{array}\right) .
$$

The SU(2) method is exact and reduces the problem to solving the ODE's for $g_{j}$.

The spirit of the RWA is to retain only those terms in the Hamiltonian that correspond to the resonance frequency. We first separate out the fast phase factors from the wavefunction:

$$
|\phi(y, t)\rangle=c_{k}(t) e^{-i \rho_{k}(t)}\left|\phi_{k}(y)\right\rangle+c_{n}(t) e^{-i \rho_{n}(t)}\left|\phi_{n}(y)\right\rangle,
$$

with $\rho_{i}(t) \equiv E_{i} \int_{0}^{t} \alpha^{2}\left(t^{\prime}\right) d t^{\prime} / \hbar$. Substituting Eq. 11 in the Schrödinger equation, we have

$$
\left(\begin{array}{c}
\dot{c_{k}} \\
\dot{c_{n}}
\end{array}\right)=\eta_{n k}\left(\begin{array}{cc}
0 & -W^{*}(t) \\
W(t) & 0
\end{array}\right)\left(\begin{array}{l}
c_{k} \\
c_{n}
\end{array}\right)
$$

where

$$
W(t) \equiv \frac{\dot{R}(t)}{R(t)} \exp \left[i \omega_{n k} \int_{0}^{t} \alpha^{2}\left(t^{\prime}\right) d t^{\prime}\right]
$$

and

$$
\eta_{n k} \equiv\left\langle\phi_{n}\left|\left(y \frac{\partial}{\partial y}+\xi\right)\right| \phi_{k}\right\rangle
$$

is a constant that depends only on the states involved.

As $\epsilon$ is small, we can expand $W(t)$ as a series of $\epsilon$. Retaining all terms up to third-order, we have:

$$
\begin{aligned}
W(t) & =\epsilon e^{i \omega_{n k} t}\left\{\omega \cos \omega t+\epsilon\left[i \omega_{n k}(\cos 2 \omega t+1)-\frac{\omega}{2} \sin 2 \omega t\right]\right. \\
& +\frac{\epsilon^{2}}{4 \omega}\left[\left(\omega^{2}-6 \omega_{n k}^{2}\right) \cos \omega t-\left(\omega^{2}+2 \omega_{n k}^{2}\right) \cos 3 \omega t\right. \\
& \left.\left.-i \frac{7}{2} \omega_{n k} \omega(\sin \omega t+\sin 3 \omega t)\right]\right\} .
\end{aligned}
$$

Note that $W(t)$ consists of oscillatory terms with various frequencies depending on $\omega$. In the spirit of RWA, we keep only terms with the lowest frequency for each $\omega$, the rationale being that $c_{k}$ and $c_{n}$ vary slowly in time and the contributions to $W$ from high frequency 
terms cancel on average over such a long time scale. It is clear then from Eq. 15 that $W$ is largest if $\omega=\omega_{n k} / N$, with $N$ an integer, because of the emergence of zero frequency terms. For these driving frequencies, $W$ is large and effective in inducing transitions, and we have resonances. At or close to a resonance, Eq. 15 simplifies tremendously, and closed-form solutions for Eq. 12 can be obtained easily.

For example, when $\delta \omega \equiv \omega-\omega_{n k} \approx 0, W(t) \approx \epsilon \omega \exp (-i \delta \omega t / 2) / 2$, and we have Rabi oscillations [15]:

$$
\begin{aligned}
& c_{k}(t)=e^{i \delta \omega t / 2}[\cos \chi t-i \delta \omega \sin \chi t /(2 \chi)], \\
& c_{n}(t)=\Gamma e^{-i \delta \omega t / 2} \sin \chi t / \chi
\end{aligned}
$$

where we took $c_{k}(0)=1, c_{n}(0)=0, \chi \equiv \sqrt{\Gamma^{2}+\delta \omega^{2} / 4}$, and $\Gamma=\Gamma_{1} \equiv \eta_{n k} \epsilon \omega / 2$. The maximum value of $\left|c_{n}\right|^{2},\left|c_{n}\right|_{\max }^{2}(\omega)=1 /\left[1+(\delta \omega / 2 \Gamma)^{2}\right]$, is a Lorentzian with a FWHM of $4 \Gamma$.

The solutions for higher $N$ resonances differ only in the widths $\Gamma=\Gamma_{N} \equiv \epsilon^{N} \eta_{n k} \gamma_{N} / 2 N$, where

$$
\gamma_{N}=\left\{\begin{array}{cc}
\left(\omega_{n k}+\delta \omega\right) & N=1 \\
\left(3 \omega_{n k}-\delta \omega\right) / 4 & N=2 \\
\frac{17 \omega_{n k}^{2}-17 \omega_{n k} \delta \omega+2 \delta \omega^{2}}{24\left(\omega_{n k}+\delta \omega\right)} & N=3
\end{array}\right.
$$

with $\delta \omega \equiv N \omega-\omega_{n k}$. The FWHM of the resonances $4 \Gamma / N \propto \epsilon^{N}$ are narrower for larger $N$, and the oscillation periods $T \propto \epsilon^{-N}$ are longer.

The energy of the system is given in RWA by

$$
E(t) \approx \alpha^{2}(t)\left[E_{k} \cos ^{2} \chi t+\left(A+E_{n}\right) \sin ^{2} \chi t\right]
$$

where $A \equiv\left[E_{k} \delta \omega^{2}+4 E_{n}\left(\Gamma^{2}-\chi^{2}\right)\right] / 4 \chi^{2}$. The energy of the particle therefore exhibits fast but small oscillations due to the $\alpha^{2}$ factor, modulated by a large but slow oscillation of period $\pi / \chi$. It follows that

$$
\frac{(1-\epsilon)^{2} E_{\max }-E_{k}}{E_{n}-E_{k}} \approx \frac{1}{1+(\delta \omega / 2 \Gamma)^{2}}=\left|c_{n}\right|_{\max }^{2}(\omega),
$$

which is again a universal Lorentzian for all resonances.

The RWA results are virtually identical with those of the $\mathrm{SU}(2)$ method. Fig. 1 can be understood completely in terms of overlapping series of the $\omega_{n k} / N$ resonances, and the positions of the peaks are in almost exact agreement with the RWA or SU(2) predictions. The resonance line shapes are well fitted by the Lorentzian Eq. 20, though the widths are underpredicted for the $N=2,3$ resonances, as shown in Table 1. For example, the $(N=2, n=4)$ resonance has a much larger width than predicted by RWA and $\mathrm{SU}(2)$, which is mainly due to the involvement of other states during transition, such as a $(1 \rightarrow 3 \rightarrow 2)$ process, which is second order in perturbation theory and so affects the $N>1$ transitions more severely. Occasionally, resonances at similar frequencies may overlap and lead to broadened widths.

At a resonance, the Rabi oscillations of the particle as predicted in RWA (Eq. 19) can be seen explicitly in Fig. 2, where the energy of the particle vs. time is shown for $n=4,3,4$ 
and $N=1,2,3$. The RWA also predicts that at exact resonances, $\chi=\Gamma$, and therefore the oscillation periods should be inversely proportional to the widths of the resonances, $T=\pi / \chi=\pi / \Gamma$. As shown in the last column of Table 1, this is clearly borne out in the numerical data, where $T \Gamma / \pi$ is listed and is found to be closed to 1 for all resonances, deviating by less than $10 \%$ for even those with strong mixing.

Using the RWA and two-level approximation, we can calculate the dynamical phase easily. Removing the dynamical phase with the help of Eq. 19, we have

$$
|\tilde{\phi}(t)\rangle \approx e^{i \omega_{n k}^{\prime} \int_{0}^{t} \sin ^{2}\left(\chi t^{\prime}\right) d t^{\prime}}\left(\begin{array}{c}
c_{k}(t) \\
c_{n}(t) e^{-i \omega_{n k} t}
\end{array}\right),
$$

where $\omega_{n k}^{\prime} \equiv A / \hbar+\omega_{n k}$. For $\omega=\omega_{n k} / N$ resonances, if we choose $t-t_{1}$ to be an integral multiple, $q$, of the cavity oscillation period $\tau$, we get

$$
\beta\left(t_{1}, t_{1}+q \tau\right)=\frac{\omega_{n k}^{\prime}}{2 \chi}\left[\tilde{\chi}-\sin \tilde{\chi} \cos \left(2 \chi t_{1}+\tilde{\chi}\right)\right],
$$

where $\tilde{\chi} \equiv \chi q \tau$. In particular, if $t_{1}=0$,

$$
\beta_{0}(q \tau) \equiv \beta(0, q \tau)=\left\{\begin{array}{cl}
\frac{\Omega(q \tau)}{2}, & \text { for }\left(2 m-\frac{1}{2}\right)<t / T \leq\left(2 m+\frac{1}{2}\right) \\
\frac{\Omega(q \tau)}{2} \pm \pi, & \text { for }\left(2 m+\frac{1}{2}\right)<t / T \leq\left(2 m+\frac{3}{2}\right)
\end{array}\right.
$$

where $\Omega(t)=\omega_{n k} t-\frac{\omega_{n k}}{2 \chi} \sin 2 \chi t$, with $m=0,1,2, \ldots$. There are sudden approximate $\pi$ jumps in $\beta_{0}$ at $t / T=(2 m+1) / 2$, as shown for example in Fig. 3 for $\tilde{\omega}=12.344$. Since $\chi \tau<<1$, the phase change in each cycle $(q=1)$ is

$$
\beta_{1}\left(t_{1}\right) \equiv \beta\left(t_{1}, t_{1}+\tau\right) \approx \omega_{n k}^{\prime} \tau \sin ^{2} \chi\left(t_{1}+\tau / 2\right)
$$

At an exact $N^{\text {th }}$ resonance, $\delta \omega=0$ and $A=0$, and so

$$
\beta_{1}\left(t_{1}\right) \approx 2 N \pi \sin ^{2}\left[\chi\left(t_{1}+N \pi / \omega_{n k}\right)\right] .
$$

Therefore, the geometric phase oscillates with an amplitude of $2 N \pi$ and period of $T$. Both of these RWA predictions are in excellent agreement with the numerical data, as shown in Fig. 2. Note that the $\pi$-jumps and the functional forms of the geometric phases are independent of $\epsilon$ and $\hbar$, as long as they are nonzero.

\section{AN ELECTRON IN A MAGNETIC FIELD}

To gain more insight into the geometric phase for a two-level system, we have studied a simple model of a magnetic field rotating around a spin-1/2 particle. Suppose an electron of charge $-e$ and mass $m$ is placed at the origin, in the presence of a magnetic field

$$
\mathbf{B}(t)=B_{0}[\sin \alpha \cos (\omega t) \hat{i}+\sin \alpha \sin (\omega t) \hat{j}+\cos \alpha \hat{k}]
$$

which has a constant magnitude $B_{0}$ but its direction sweeps out a cone with an opening angle $\alpha, 0<\alpha<\pi$, at a constant angular speed $\omega$. The Hamiltonian of the system is given by 


$$
H(t)=\frac{e}{m} \mathbf{B} \cdot \mathbf{S}=-\frac{\hbar \omega_{1}}{2}\left(\begin{array}{cc}
\cos \alpha & e^{-i \omega t} \sin \alpha \\
e^{i \omega t} \sin \alpha & -\cos \alpha
\end{array}\right),
$$

where $\omega_{1} \equiv-e B_{0} / m$ and $\mathbf{S}$ is the spin matrix.

The system can be solved analytically [16]. The instantaneous eigenspinors of $H(t)$ with eigenenergies $E_{+}=-E_{-}=-\hbar \omega_{1} / 2$ respectively are

$$
\left|\psi_{+}(t)\right\rangle=\left(\begin{array}{c}
\cos (\alpha / 2) \\
e^{i \omega t} \sin (\alpha / 2)
\end{array}\right)
$$

and

$$
\left|\psi_{-}(t)\right\rangle=\left(\begin{array}{c}
\sin (\alpha / 2) \\
-e^{i \omega t} \cos (\alpha / 2)
\end{array}\right)
$$

Suppose the electron spin is initially parallel to $\mathbf{B}(0)$, and we consider the case when the particle makes a transition to spin down along the instantaneous direction of $\mathbf{B}$. This happens with unit probability if $\omega=-\omega_{1} / \cos \alpha$, provided that $\cos \alpha \neq 0$. The state vector at any time is then $[16]$

$$
|\psi(t)\rangle=e^{-i \omega t / 2}\left\{\cos \left(\frac{\lambda t}{2}\right)\left|\psi_{+}(t)\right\rangle+i \sin \left(\frac{\lambda t}{2}\right)\left|\psi_{-}(t)\right\rangle\right\},
$$

where $\lambda=\omega \sin \alpha$.

The Pancharatnam phases comparing the initial state with the state at time $t$ for different values of $\alpha$ are shown in Fig. (4-6). The sudden $\pi$-jump and a two-period oscillation can be seen in the case when $\alpha=1 / p, p$ being an even integer, such as the case in Fig. (4). When $p$ is an odd integer, the Pancharatnam phase performs a single-period oscillation with no $\pi$-jump, as shown in Fig. (5). It has neither single-period nor two-period oscillation when other values of $\alpha$ are used, Fig. (6). If $t=q \tau$, with $q$ an integer and $\tau=2 \pi / \omega$,

$$
\beta_{0}(q \tau)=\left\{\begin{array}{cl}
-\frac{\Omega(q \tau)}{2}, & \text { for }\left(2 m-\frac{1}{2}\right)<q \tau / T \leq\left(2 m+\frac{1}{2}\right) \\
-\frac{\Omega(q \tau)}{2} \pm \pi, & \text { for }\left(2 m+\frac{1}{2}\right)<q \tau / T \leq\left(2 m+\frac{3}{2}\right)
\end{array}\right.
$$

which has the same form as Eq. 23, where $m$ is an integer, $T=2 \pi / \lambda$, and

$$
\Omega(t) \equiv\left(\frac{\omega_{1}}{\lambda} \sin \lambda t+\omega t\right) .
$$

For the case $t_{1}=(q-1) \tau$ and $t=q \tau$,

$$
\beta_{1}\left(t_{1}\right)=\left\{\begin{aligned}
-\frac{1}{2}\left[\Omega\left(t_{1}+\tau\right)-\Omega\left(t_{1}\right)\right], & \text { for } 0<\sin \alpha<1 / 2 \\
-\frac{1}{2}\left[\Omega\left(t_{1}+\tau\right)-\Omega\left(t_{1}\right)\right] \pm \pi, & \text { for } 1 / 2<\sin \alpha<1
\end{aligned}\right.
$$

In the limit $\alpha \ll 1$,

$$
\beta_{1}\left(t_{1}\right) \approx-2 \pi \sin ^{2}\left(\frac{\lambda}{2}\left(t_{1}+\tau / 2\right)\right)
$$


which has the same form as Eq. 25.

In the cyclic limit, i.e., $t=q \tau=T \equiv 2 \pi / \lambda$,

$$
\Omega(T)=\omega T=\omega q \tau=2 q \pi
$$

and thus the geometric phases are $\beta_{0}(T)=-(q-1) \pi$ and $\beta_{1}(T)=-\pi$.

Since the geometric phases in the two models - an electron in a rotating magnetic field and a particle in a vibrating cavity - are remarkably similar, we conjecture that the main features of the generalized geometric phase we calculated, especially the $\pi$-jumps, are universal for a particle in transition from one state to another. Similar features about the geometric phase have also been obtained in [17].

\section{GEOMETRICAL MODEL}

Here we present a geometrical model that helps to visualize the evolution of the geometric phase of a two-level system. It is clear from Eq. 30 that the state vector traces out a path on the unit sphere defined by the angular coordinates $\theta \equiv \lambda t$ and $\phi \equiv \omega t$. Note that we have used a time-dependent basis $\left|\psi_{+}(t)\right\rangle,\left|\psi_{-}(t)\right\rangle$, which mark the North and South Poles respectively on the unit sphere. The excitation condition of our system therefore gives us the trajectory of the state on this unit sphere,

$$
\theta=\phi \sin \alpha
$$

which is a spiral curve.

The solid angle subtended at the origin by the spiral curve and the geodesic, $\phi=0$, up to any $t=q \tau$ is

$$
\begin{aligned}
\Omega_{o} & =\int_{0}^{2 q \pi} \int_{0}^{\phi^{\prime} \sin \alpha} \sin \theta^{\prime} d \theta^{\prime} d \phi^{\prime} \\
& =2 q \pi-\frac{\sin (2 q \pi \sin \alpha)}{\sin \alpha},
\end{aligned}
$$

which coincides with Eq. 32, in the limit $\alpha \ll 1$. Therefore, we see from Eq. 31 and Eq. 33 that the geometric phases $\beta_{0}$ and $\beta_{1}$ are simply the solid angles subtended by the spirial curve, $\theta=\alpha \phi$, from $\phi=0$ and $\phi=2(q-1) \pi$ up to $\phi=2 q \pi$ respectively, divided by -2 . This picture is a generalization of Berry's [6] for adiabatic and cyclic evolution corresponding to trajectories with constant $\theta=\theta_{0}$, and the solid angle is simply $\Omega_{o}=2 \pi\left(1-\cos \theta_{0}\right)$. Even the $\pi$-jump can be represented in this model by a jump of the particle from one sphere to another if its trajectory happens to reach the South Pole, which depends on the value of the angle $\alpha$. The double-sphere picture reminds one of the underlying $\mathrm{SU}(2)$ structure.

\section{SUMMARY}

In summary, we have reported the first calculation of the quantum geometric phase of a physical system in resonance - that of a particle in a vibrating cylindrical or spherical cavity, and we have shown that it acquires sudden $\pi$-jumps when the particle makes transitions from 
one state to another. We have derived analytic expressions in the RWA and SU(2) methods, which give excellent description of the energy, wavefunctions, and the geometric phases at these resonances. We found remarkably similar properties of the geometric phases for the simple system of an electron in a rotating magnetic field, which led us to conjecture that the main features of the generalized geometric phases and especially the $\pi$-jump we found are universal. We have also developed a geometrical model to help visualize these phases.

This work is partially supported by a Chinese University Direct Grant (Project ID: 2060093). 


\section{REFERENCES}

[1] P. Hasenfratz and J. Kuti, Phys. Rep. 40, 75 (1978); K. Colanero and M.-C. Chu, Phys. Rev. C 65, 045203 (2001); K. Colanero, Phd thesis, Chinese University, 2001.

[2] B.P. Barber et al., Phys. Rep. 281, 65 (1997).

[3] G.T. Moore, J. Math. Phys. 11, 2679 (1970); P.W. Milonni, The Quantum Vacuum (Academic Press, New York, 1993); N.D. Birrell and P.C.W. Davies, Quantum Fields in Curved Space (Cambridge University Press, Cambridge, 1982).

[4] S.W. Hawking, Nature 248, 30 (1974); Commun. Math. Phys. 43, 199 (1975).

[5] For example, C.K. Law, Phys. Rev. Lett. 73, 1931 (1994); V.V. Dodonov and A.B. Klimov, Phys. Rev. A 53, 2664 (1996), and references therein; A. Lambrecht, M.T. Jaekel and S. Reynaud, Phys. Rev. Lett. 77, 615 (1996); C.K. Cole and W.C. Schieve, Phys. Rev. A 52, 4405 (1995); P. Meystre et al., J. Opt. Soc. Am. B 2, 1830 (1985); Ying Wu et al., Phys. Rev. A 59, 3032 (1998); Ying Wu et al., Phys. Rev. A 59, 1662 (1998); K.W. Chan, Master Thesis, The Chinese University of Hong Kong (unpublished), 1999; K. Colanero and M.-C. Chu, Phys. Rev. E 62, 8663 (2000).

[6] M.V. Berry, Proc. Roy. Soc. Lond. A 392, 45 (1984).

[7] A. Shapere and F. Wilczek, Geometric Phase in Physics (World Scientific, Singapore, 1989).

[8] Y. Aharonov and J. Anandan, Phys. Rev. Lett. 58, 1593 (1987).

[9] D.J. Moore, Phys. Rep. 210, 1 (1991).

[10] D.M. Bird and A.R. Preston, Phys. Rev. Lett. 61, 2863 (1988).

[11] A.G. Wagh and V.C. Rakhecha, Phys. Lett. A 148, 17 (1990) .

[12] K. Colanero and M.-C. Chu, Phys. Rev. A 60, 1845 (1999).

[13] J. Samuel and R. Bhandari, Phys. Rev. Lett. 60, 2339 (1988).

[14] C. M. Cheng and P. C. W. Fung, J. Phys. A: Math. Gen. 22, 3493 (1989); K.M. Cheng and P.C.W. Fung, J. Phys. A: Math. Gen. 23, 4851 (1990).

[15] J.J. Sakurai, Modern Quantum Mechanics (Addison-Wesley, Redwood City, 1985).

[16] David J. Griffiths, Introduction to Quantum Mechanics (Prentice Hall, Upper Saddle River, 1995).

[17] R. Bhandari, Phys. Rev. Lett. 88, 10 (2002). 


\section{FIGURES}

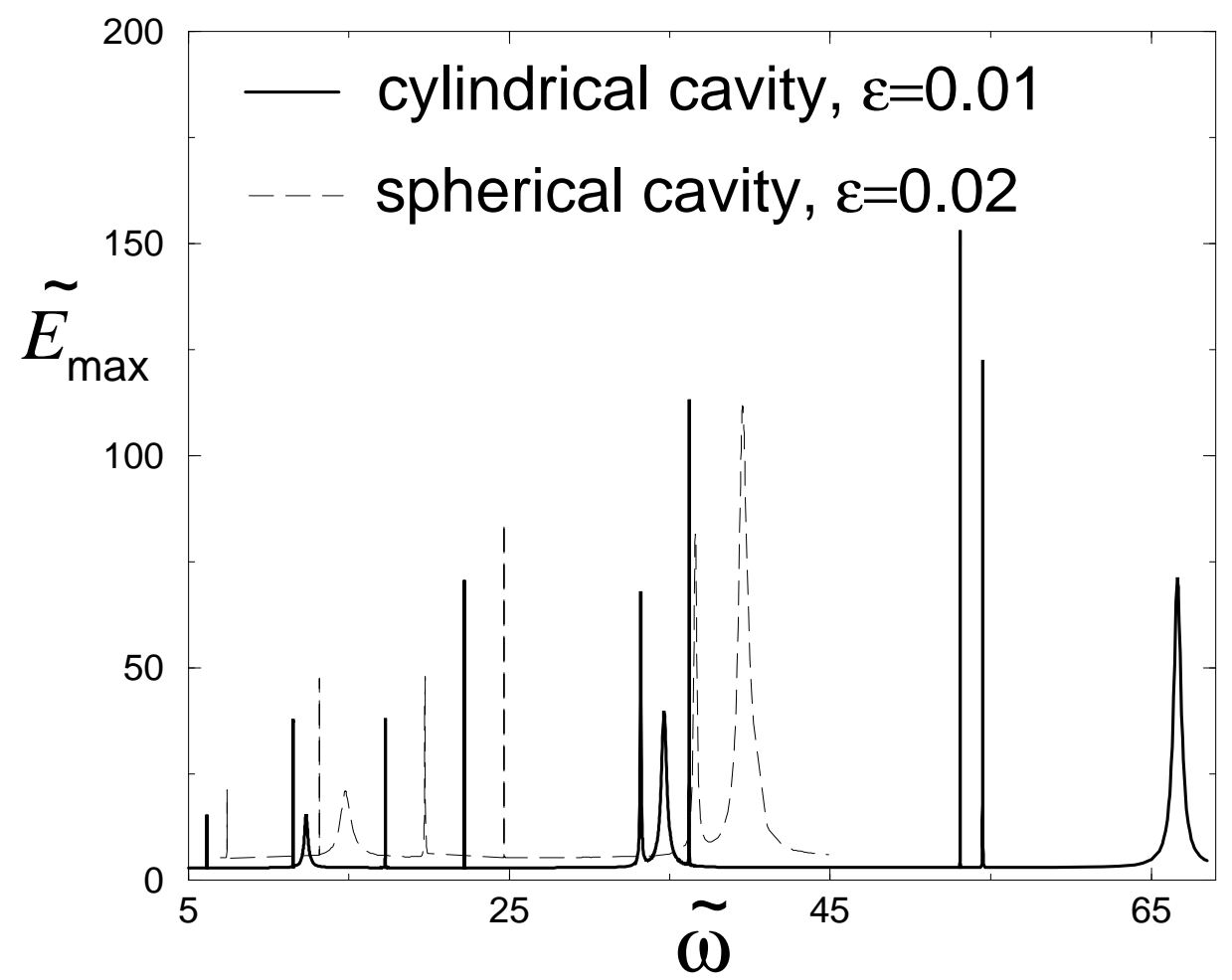

FIG. 1. The maximum energy of the particle as a function of the driving frequency, obtained by solving the Schrödinger equation numerically, for a cylindrical cavity with $\epsilon=0.01$ (solid line) and a spherical cavity with $\epsilon=0.02$ (dashed line).

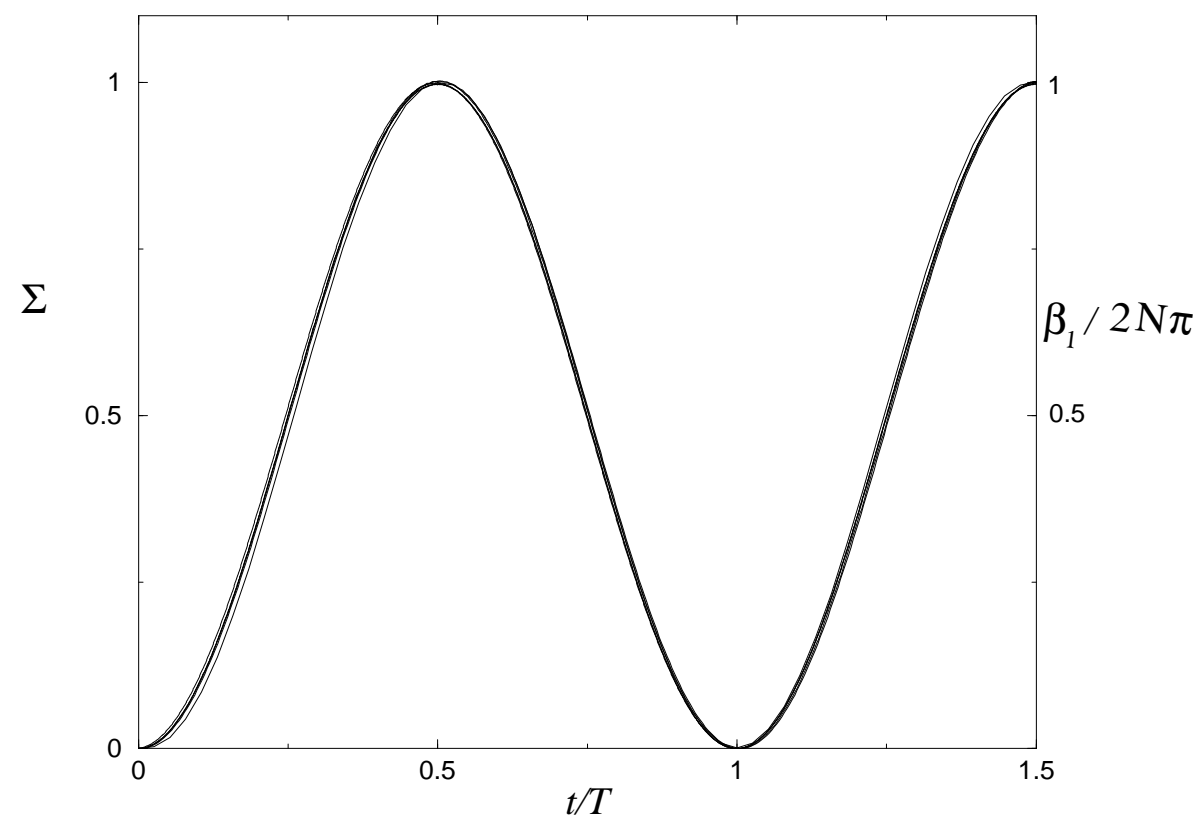


FIG. 2. The scaled energy, $\Sigma \equiv\left(E \alpha^{-2}-E_{k}\right) /\left(E_{n}-E_{k}\right)$ where $E_{k}$ and $E_{n}$ are the unperturbed eigenenergies, and geometric phase acquired at each cycle, $\beta_{1} / 2 N \pi$, vs. $t / T, T$ being the oscillation period of the geometric phase, for the case of cylindrical cavity at $\tilde{\omega}=66.632,17.278$, and 22.21227, using $\epsilon=0.01$.
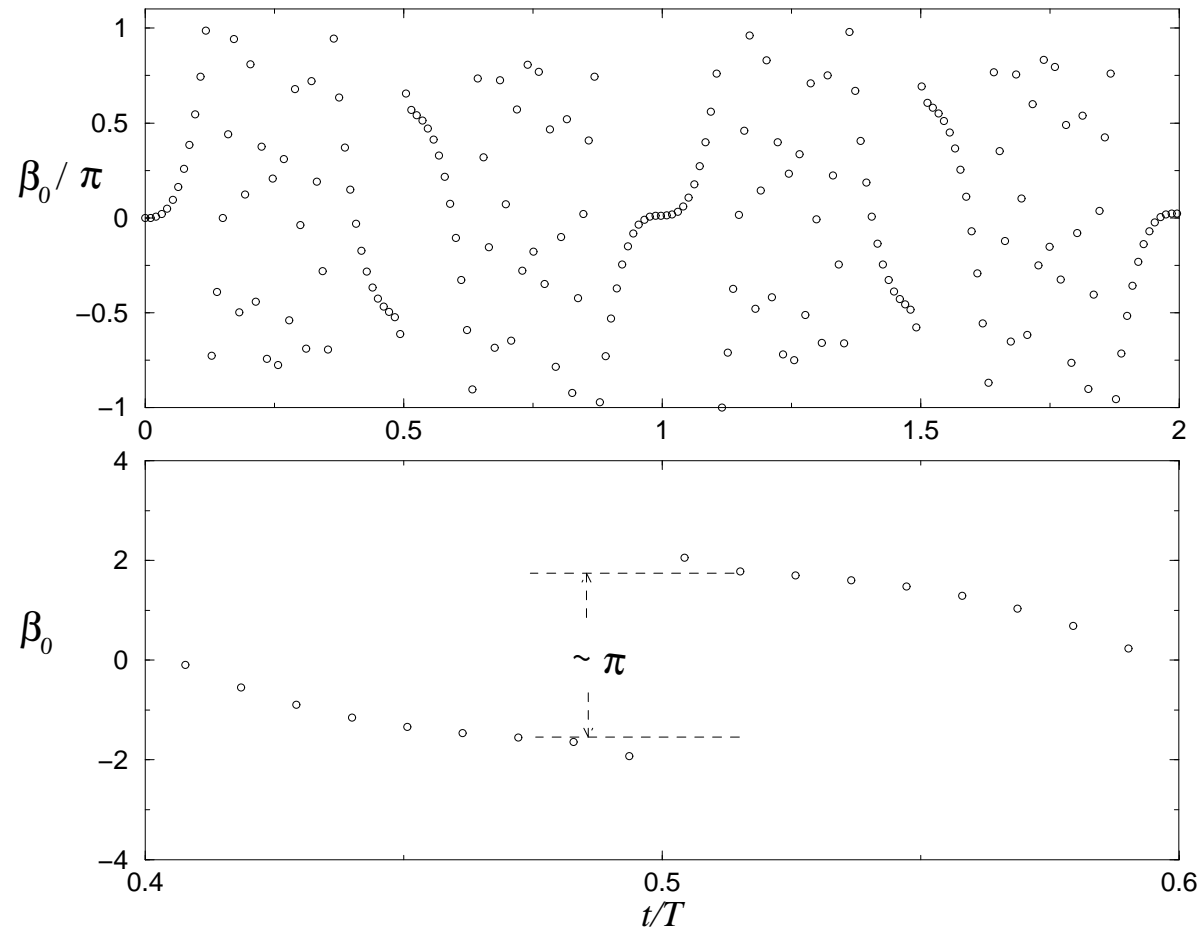

FIG. 3. $\beta_{0}$ vs. $t / T$ at $\tilde{\omega}=12.344$ for a cylindrical cavity with $\epsilon=0.01$. The region around $t / T=0.5$ is enlarged and shown in the lower panel to show the approximate $\pi$-jump. 

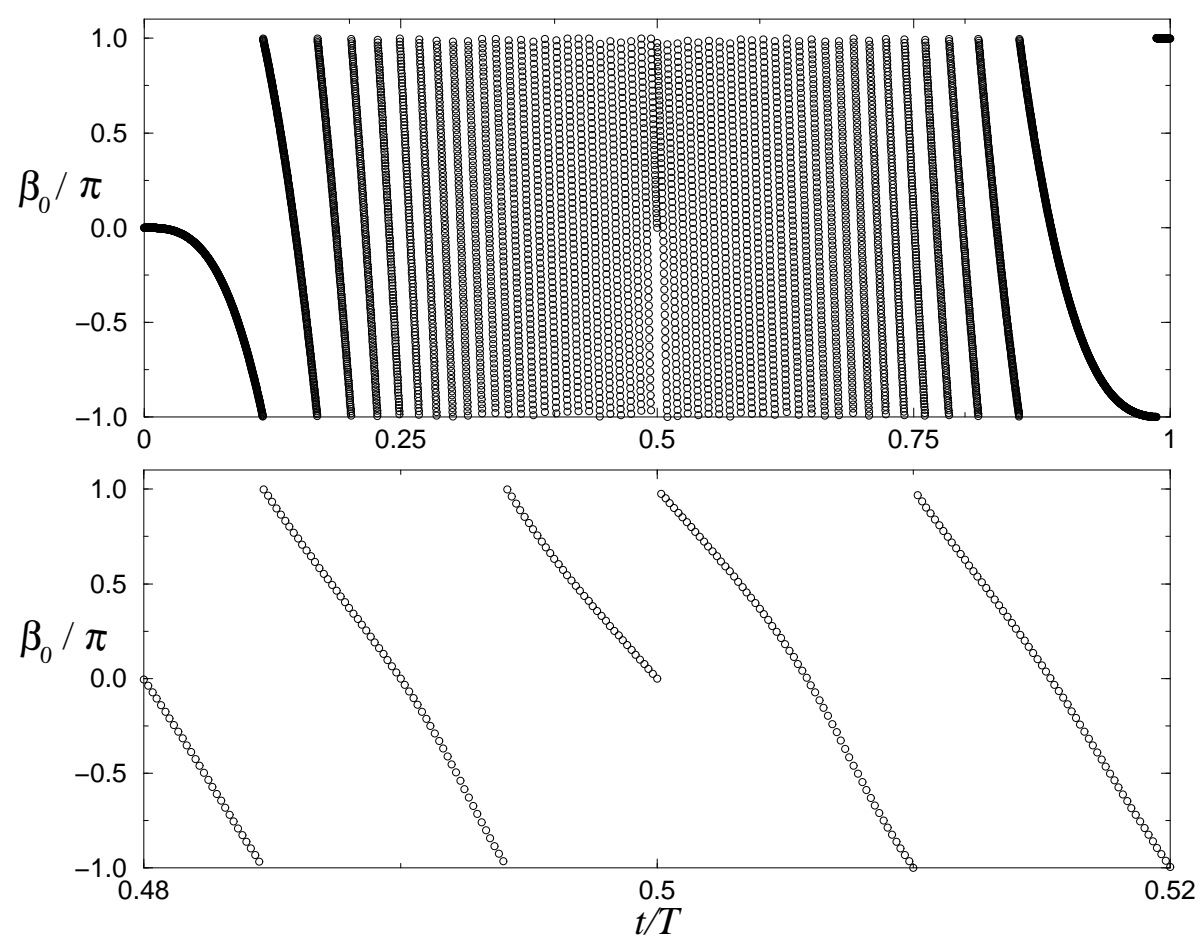

FIG. 4. Same as Fig. 3, but for a spin- $1 / 2$ particle in a rotating magnetic field, $\alpha=1 / 100$, and for $t \neq n \tau$.
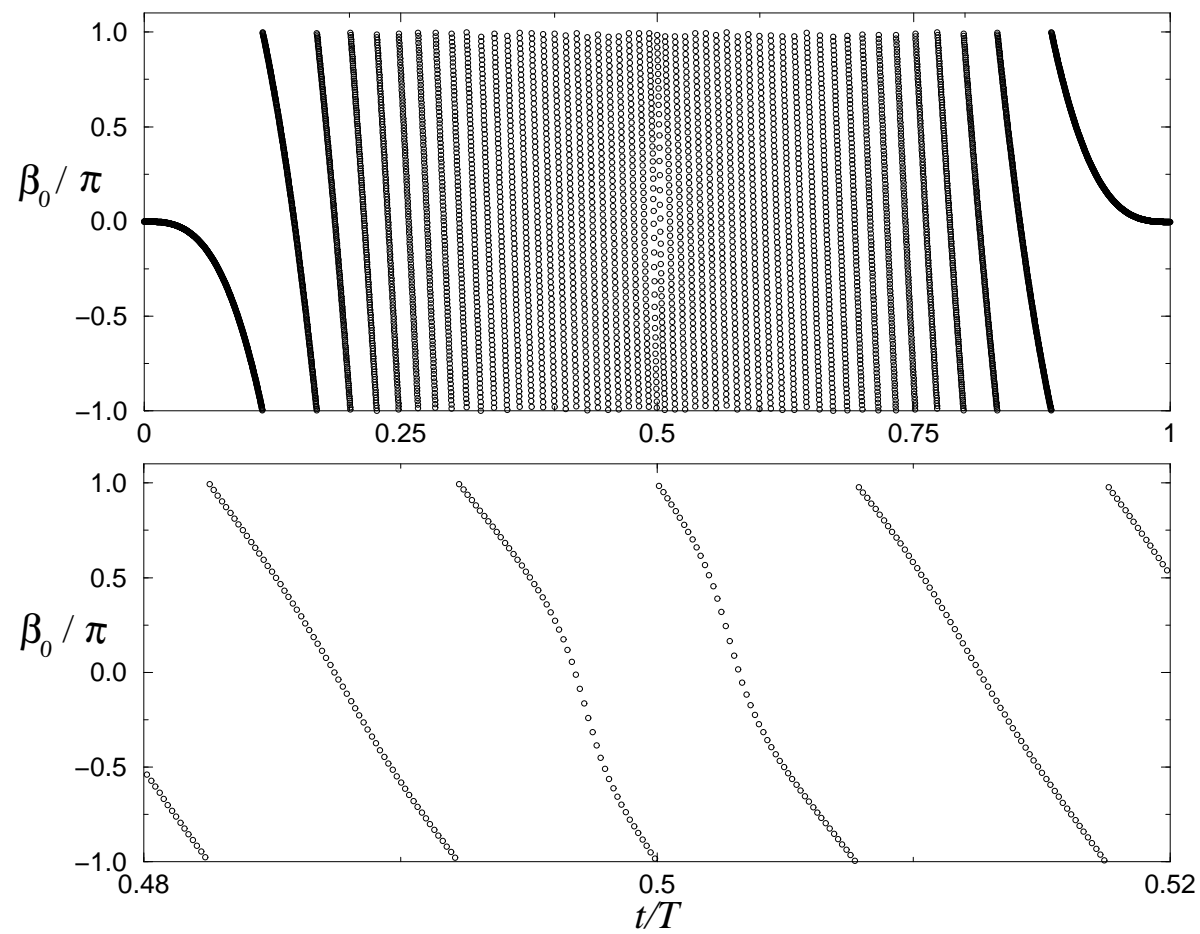

FIG. 5. Same as Fig. 4, but for $\alpha=1 / 101$. 

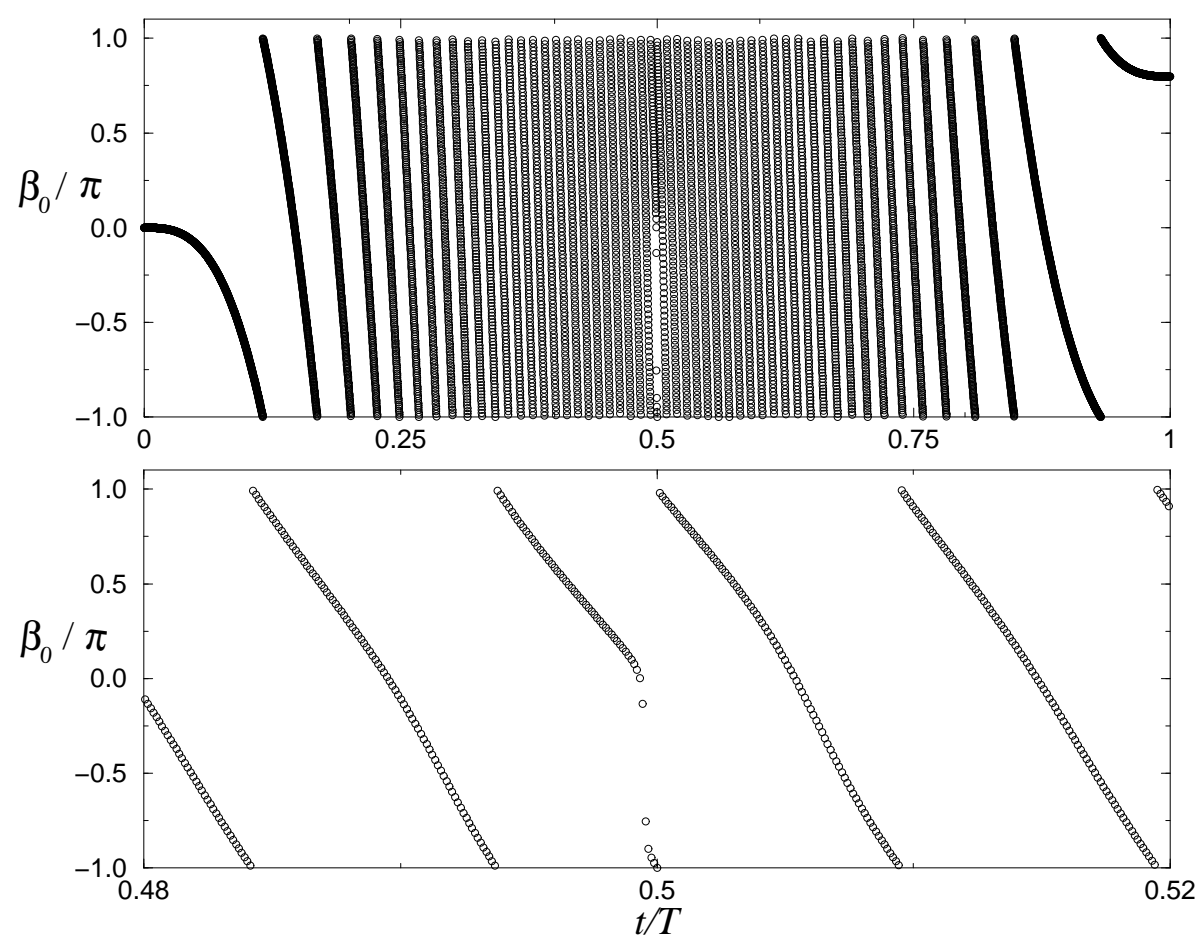

FIG. 6. Same as Fig. 4, but for $\alpha=5 / 501$. 


\section{TABLES}

\begin{tabular}{|c|c||c|c|c|}
\hline \hline \multicolumn{2}{|c||}{ Resonances } & \multicolumn{1}{c|}{$\Gamma$} & & $T \Gamma / \pi$ \\
\hline $\mathrm{N}$ & $\mathrm{n}$ & Numerical & RWA & Numerical \\
\hline 1 & 2 & $6.17(7.45)$ & $6.17(7.40)$ & $1.00(1.01)$ \\
\hline 1 & 3 & 16.6 & 17.3 & 0.98 \\
\hline 1 & 4 & 33.2 & 33.3 & 1.00 \\
\hline \hline 2 & 2 & $4.15(4.66)$ & $4.63(5.55)$ & $0.99(0.96)$ \\
\hline 2 & 3 & $50.0(76.5)$ & $13.0(14.8)$ & $1.00(1.02)$ \\
\hline 2 & 4 & $891(532)$ & $25.0(27.8)$ & $1.09(1.17)$ \\
\hline 2 & 5 & 136 & 40.7 & 0.93 \\
\hline \hline 3 & 4 & $205(341)$ & $23.6(26.2)$ & $1.02(1.07)$ \\
\hline 3 & 5 & 15700 & 38.5 & 1.02 \\
\hline 3 & 6 & 78300 & 56.8 & 1.07 \\
\hline \hline
\end{tabular}

TABLE I. Scaled widths $\left(\tilde{\Gamma} \equiv \mu R_{0}^{2} \Gamma / \hbar \epsilon^{N} \eta_{n k}\right)$ of the lowest $k=1$ resonances for cylindrical cavity with $\epsilon=0.01$. Full numerical results are compared to the RWA values. The widths multiplied by the Rabi oscillation period, both extracted from the numerical data, are also shown in the last column. Corresponding numbers for spherical cavity with $\epsilon=0.02$ are shown in parentheses. 